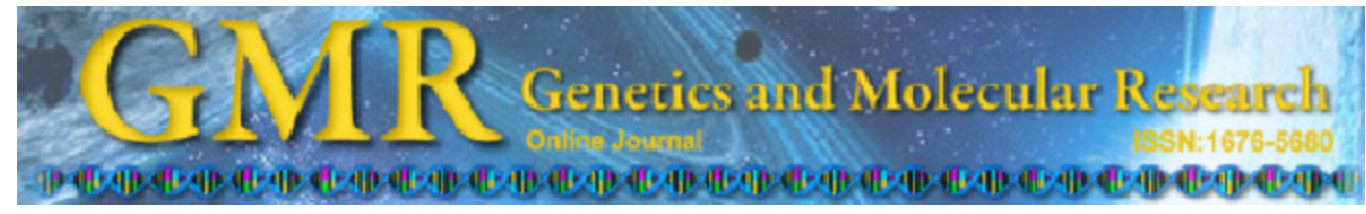

\title{
Association of vitamin D receptor BsmI gene polymorphism with risk of low bone mineral density in post-menopausal women: a meta-analysis
}

\author{
C. Ma ${ }^{1}$, Q.L. Zhou ${ }^{1}$, Y.J. Deng ${ }^{1}$, X. Liang ${ }^{2}$, D.P. Wu ${ }^{1}$ and Y.Z. Dong ${ }^{1}$ \\ ${ }^{1}$ Department of Orthopedics, \\ The First Affiliated Hospital of Xinxiang Medical College, Xinxiang, China \\ ${ }^{2}$ Department of Anesthesiology, \\ The First Affiliated Hospital of Xinxiang Medical College, Xinxiang, China \\ Corresponding author: Y.Z. Dong \\ E-mail: dongyunzhen123@163.com
}

Genet. Mol. Res. 13 (3): 7791-7799 (2014)

Received July 25, 2013

Accepted February 21, 2014

Published September 26, 2014

DOI http://dx.doi.org/10.4238/2014.September.26.17

\begin{abstract}
The vitamin D receptor BsmI gene polymorphism is reportedly associated with low bone mineral density (BMD) in postmenopausal women, but results from previous studies are conflicting. In the present study, we investigated the association between this polymorphism and the risk of low BMD through a metaanalysis of published studies. A literature search of the Pubmed, Embase, and CNKI databases from inception through July 2013 was conducted. The meta-analysis was performed using the STATA 12.0 software. Crude odds ratios with $95 \%$ confidence intervals were used to assess the strength of any association. Eleven case-control studies were included for a total of 1468 low BMD cases and 2177 healthy controls. No significant variation in low BMD risk was detected in any of the genetic models. Further stratified analyses were performed to examine the effect of ethnicity. In the subgroup analysis, no significant
\end{abstract}


association was found in Caucasians and in Asians. The meta-analysis results suggest that the $B s m I$ polymorphism is not associated with low BMD risk in postmenopausal women.

Key words: Vitamin D receptor; BsmI; Gene polymorphism; Bone mineral density; Meta-analysis

\section{INTRODUCTION}

Osteoporosis is a common health problem, particularly in postmenopausal women, and is characterized by decreased bone mineral density (BMD) and deterioration of skeletal microarchitecture, leading to increased bone fragility and fracture risk (Kanis et al., 1994). Epidemiological studies have shown that BMD is influenced by many environmental factors, such as smoke exposure, waist to hip ratio, physical activity, and body mass index (Seo et al., 2008). In addition, many studies have shown that genetic factors play an important role in the pathogenesis of BMD. Evidence is accumulating that up to $80 \%$ of the heritability for BMD in the general population can be attributed to genetic factors (Riggs, 1997).

Vitamin D plays a crucial role in BMD metabolism via the vitamin D receptor (VDR), which is a member of the nuclear receptor family of transcription factors (Moore et al., 2006). VDR plays a role in regulating calcium homoeostasis through binding and nuclear translocation of 1- $\alpha, 25$-dihydroxyvitamin $\mathrm{D}_{3}$, affecting bone resorption and increasing calcium absorption (Pike et al., 2002). In recent years, candidate gene association studies have explored the association between BMD and the VDR gene, which is located on chromosome 12cen-q12 and contains 14 exons. Morrison et al. (1994) first reported that VDR gene polymorphisms could predict spinal and femoral BMD in Caucasian women. Since then, a large number of studies regarding the association between VDR polymorphisms and BMD have been published, including studies examining the FokI, BsmI, and $A p a \mathrm{I}$ variants.

The Bsm I polymorphism is the most important subtype of the VDR gene polymorphisms. Several association studies have reported conflicting results of the relationship between the Bsm I genetic polymorphism and BMD risk. Furthermore, Qin et al. (2013) performed a meta-analysis of all studies relating the BsmI polymorphism with low BMD risk and found that it was not associated with low BMD in the overall population examined. However, they did not perform a subgroup analysis aimed at postmenopausal women. Osteoporosis is a major health problem in women after menopause. Remodeling imbalance plays an important role in the pathogenesis of postmenopausal osteoporosis (Turner et al., 1994). Decreased activity of the enzyme 1- $\alpha, 25$-dihydroxyvitamin $\mathrm{D}_{3}$ hydroxylase, which converts $25-\mathrm{OH}$ vitamin $\mathrm{D}$ into $1,25-(\mathrm{OH})_{2}$ vitamin $\mathrm{D}$ (potent vitamin D), has been reported after menopause due to estrogen deficiency (Gallagher et al., 2001). In this study, we performed a meta-analysis to assess the association between the Bsm I polymorphism of the VDR gene and low BMD in postmenopausal women.

\section{MATERIAL AND METHODS}

\section{Literature search}

Two reviewers searched the Pubmed, Embase, and CNKI databases to retrieve pa- 
pers linking the BsmI polymorphism and low BMD risk that were available up to July 2013 without language restrictions using the following key words: "vitamin D receptor", "BsmI", "bone mineral density", "polymorphism", "single nucleotide polymorphism", "genetic polymorphism", and "BMD". References in the eligible articles or textbooks were also reviewed manually to identify other studies containing these key words. Any disagreement was resolved by discussion between the researchers.

\section{Inclusion and exclusion criteria}

The following inclusion criteria were used to select studies: i) case-control studies that addressed low BMD cases and healthy controls; ii) studies that evaluated the association between the $B s m$ I polymorphism and low BMD risk; and iii) studies that included sufficient data for extraction. Studies were excluded when: i) they were not case-control studies that evaluated the association between the BsmI polymorphism and low BMD risk; ii) the articles were case reports, letters, reviews, meta-analyses, or editorials; iii) the studies were based on incomplete data, no usable data were reported, or duplicate data.

\section{Data extraction}

From each eligible report, we recorded the last name of the first author, year of publication, country of the study and ethnicity, numbers of cases and controls, gene polymorphisms, and evidence of Hardy-Weinberg equilibrium (HWE) in controls. Two investigators (Bizeng Zhao and Wei Zhang) extracted the data independently, and the results were reviewed by a third investigator (Zubin Zhou).

\section{Statistical analysis}

The strength of the association between the BsmI polymorphism and low BMD risk was measured by odds ratios (ORs) with $95 \%$ confidence intervals (CIs) under 4 genetic models, including a homozygote comparison ( $\mathrm{BB} v s \mathrm{bb}$ ), a heterozygote comparison $(\mathrm{BB} v s \mathrm{Bb})$, a dominant model $(\mathrm{Bb}+\mathrm{bb} v s \mathrm{BB})$, and a recessive model $(\mathrm{BB}+\mathrm{Bb} v s \mathrm{bb})$ between groups. Heterogeneity was quantified using the $I^{2}$ test: $I^{2}<25 \%$, no heterogeneity; $I^{2}=25-50 \%$, moderate heterogeneity; $I^{2}=50-75 \%$, high heterogeneity, $I^{2}>75 \%$, extreme heterogeneity. When the effects were assumed to be homogeneous $\left(I^{2}>50 \%\right)$, the fixed-effects model was used. Otherwise, the random-effects model was more appropriate. Subgroup analysis based on ethnicity was used to explore and explain the diversity among the results of different studies. Begg's funnel plot was used to assess publication bias $(\mathrm{P}<0.05$ was considered to be statistically significant). Sensitivity analysis was performed by removing meta-analysis studies individually, excluding those whose genotype distributions of the control groups deviated from HWE. We assessed HWE in controls for each study using the $\chi^{2}$ test and $\mathrm{P}<0.05$ was considered to be significant disequilibrium. All analyses were conducted using the STATA Version 12.0 software (Stata Corp; College Station, TX, USA). 


\section{RESULTS}

\section{Studies included in the meta-analysis}

According to our inclusion criteria, a total of 11 eligible studies involving 1468 cases and 2177 controls were included in the pooled analysis (Berg et al., 1996; Vandevyver et al., 1997; Gomez et al., 1999; Valimaki et al., 2001; Zajickova et al., 2002; Douroudis et al., 2003; Zhu et al., 2004; Garnero et al., 2005; Horst-Sikorska et al., 2005; Mitra et al., 2006; Ge et al., 2009). The characteristics of selected studies are summarized in Figure 1. Genotyping in all assays was conducted using restriction fragment length polymorphism analysis. All studies were case-control studies that evaluated the association between the BsmI polymorphism and low BMD risk. All the articles were written in English except for Ge et al. (2009) and Zhu et al. (2004). There were 3 studies from Asian countries and 8 studies from European countries. The publishing year of the included studies ranged from 1996 to 2009. An HWE test was conducted on genotype distribution of the controls in all included studies; all analyzed HWE except for 3 studies (Douroudis et al., 2003; Zhu et al., 2004; Ge et al., 2009). The baseline characteristics of all studies included are summarized in Table 1.

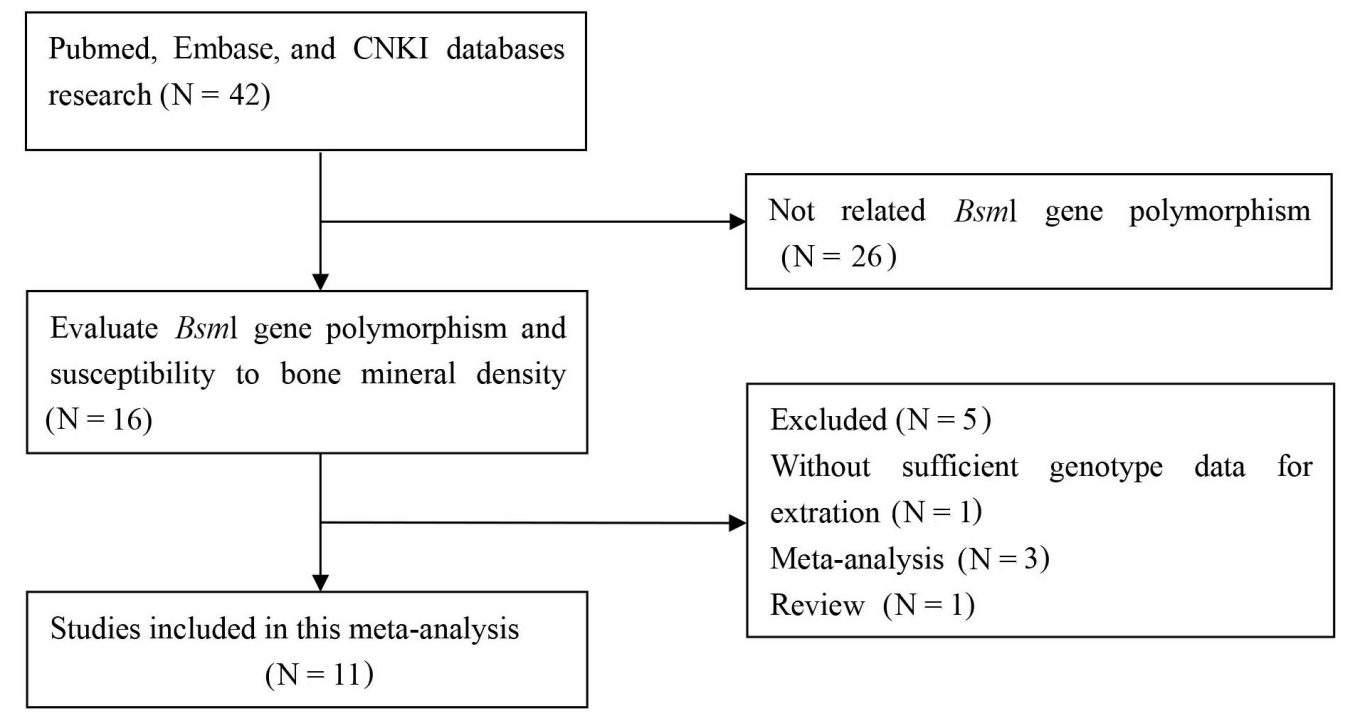

Figure 1. Flow diagram of study searching and selection process.

\section{Quantitative synthesis}

A summary of the meta-analysis findings for the association between the BsmI polymorphism and low BMD risk is shown in Table 2 and Figure 2. We found no significant association between $B s m \mathrm{I}$ polymorphisms and low BMD risk in any of the genetic models (BB $v s$ bb: $\mathrm{OR}=0.78,95 \% \mathrm{CI}=0.42-1.45$; $\mathrm{BB} v s \mathrm{Bb}: \mathrm{OR}=0.91,95 \% \mathrm{CI}=0.72-1.15$; dominant 
model: $\mathrm{OR}=1.17,95 \% \mathrm{CI}=0.75-1.81$; recessive model: $\mathrm{OR}=0.80,95 \% \mathrm{CI}=0.53-1.23)$. In the subgroup analysis by ethnicity, the studies included were divided into Asian and Caucasian populations, and no significant association was found between $B s m \mathrm{I}$ polymorphisms and low $\mathrm{BMD}$ risk in Asians $(\mathrm{BB} v s$ bb: $\mathrm{OR}=0.92,95 \% \mathrm{CI}=0.14-6.14 ; \mathrm{BB} v s \mathrm{Bb}: \mathrm{OR}=0.92$, $95 \% \mathrm{CI}=0.25-3.38$; dominant model: $\mathrm{OR}=1.03,95 \% \mathrm{CI}=0.22-4.73$; recessive model: $\mathrm{OR}=$ $0.91,95 \% \mathrm{CI}=0.31-2.68$ ) and in Caucasians (BB $v s$ bb: $\mathrm{OR}=0.78,95 \% \mathrm{CI}=0.41-1.47$; $\mathrm{BB} v s$ $\mathrm{Bb}: \mathrm{OR}=0.97,95 \% \mathrm{CI}=0.75-1.26$; dominant model: $\mathrm{OR}=1.14,95 \% \mathrm{CI}=0.75-1.73$; recessive model: $\mathrm{OR}=0.77,95 \% \mathrm{CI}=0.48-1.26$ ). Furthermore, sensitivity analysis was performed with controls in HWE and the result was not altered, suggesting that the results of this metaanalysis are stable.

Table 1. Characteristics of literatures included in the meta-analysis.

\begin{tabular}{|c|c|c|c|c|c|c|c|c|c|c|c|}
\hline \multirow[t]{2}{*}{ Study included } & \multirow[t]{2}{*}{ Year } & \multirow[t]{2}{*}{ Area } & \multirow[t]{2}{*}{ Race } & \multirow[t]{2}{*}{ Cases/Controls } & \multicolumn{3}{|c|}{ Genotypes for cases } & \multicolumn{3}{|c|}{ Genotypes for controls } & \multirow{2}{*}{$\begin{array}{c}\text { HWE } \\
\text { test }\end{array}$} \\
\hline & & & & & $\mathrm{BB}$ & $\mathrm{Bb}$ & $\mathrm{bb}$ & BB & $\mathrm{Bb}$ & $\mathrm{bb}$ & \\
\hline Berg et al. & 1996 & Norway & Caucasian & $19 / 30$ & 4 & 8 & 7 & 8 & 11 & 11 & 0.16 \\
\hline Vandevyver & 1997 & Belgium & Caucasian & $86 / 698$ & 12 & 50 & 24 & 127 & 368 & 203 & 0.08 \\
\hline Gomez et al. & 1999 & Spain & Caucasian & $76 / 236$ & 13 & 38 & 25 & 38 & 109 & 89 & 0.63 \\
\hline Valimaki et al. & 2001 & Finland & Caucasian & $372 / 111$ & 44 & 175 & 153 & 20 & 55 & 36 & 0.90 \\
\hline Zajickova et al. & 2002 & Czech Republic & Caucasian & $65 / 33$ & 21 & 24 & 20 & 10 & 13 & 10 & 0.22 \\
\hline Douroudis et al. & 2003 & Greece & Caucasian & $35 / 44$ & 3 & 12 & 20 & 10 & 29 & 5 & 0.03 \\
\hline Zhu et al. & 2004 & China & Asian & $40 / 158$ & 6 & 26 & 8 & 7 & 105 & 46 & 0.00 \\
\hline Garnero et al. & 2005 & France & Caucasian & $240 / 349$ & 50 & 124 & 66 & 40 & 162 & 147 & 0.64 \\
\hline Mitra et al. & 2006 & India & Asian & $97 / 119$ & 19 & 38 & 40 & 51 & 46 & 22 & 0.05 \\
\hline Horst-Sikorska et al. & 2005 & Poland & Caucasian & $85 / 191$ & 10 & 35 & 40 & 33 & 85 & 73 & 0.34 \\
\hline Ge et al. & 2009 & China & Asian & $353 / 208$ & 6 & 33 & 314 & 4 & 12 & 192 & 0.00 \\
\hline
\end{tabular}

Table 2. Summary ORs and 95\%CI of BsmI gene polymorphism with bone mineral density risk.

\begin{tabular}{|c|c|c|c|c|c|c|c|c|c|c|}
\hline \multirow[t]{2}{*}{ Subgroup } & \multirow[t]{2}{*}{ Genetic model } & \multicolumn{2}{|c|}{ Sample size } & \multirow[t]{2}{*}{ Type of model } & \multicolumn{2}{|c|}{ Test of heterogeneity } & \multicolumn{2}{|c|}{ Test of association } & \multicolumn{2}{|c|}{$\begin{array}{c}\text { Test of } \\
\text { publication bias }\end{array}$} \\
\hline & & Case & Control & & $\mathrm{I}^{2}$ & $\mathrm{P}$ & OR & $95 \% \mathrm{CI}$ & $\mathrm{z}$ & $P$ \\
\hline \multirow[t]{4}{*}{ Overall } & $\mathrm{BB} v s \mathrm{bb}$ & 1468 & 2177 & Random & $81.8 \%$ & 0.00 & 0.78 & $0.42-1.45$ & 0.00 & 1.00 \\
\hline & $\mathrm{BB} v s \mathrm{Bb}$ & & & Fixed & $42.8 \%$ & 0.06 & 0.91 & $0.72-1.15$ & 0.00 & 1.00 \\
\hline & Dominant model & & & Random & $71 \%$ & 0.00 & 1.17 & $0.75-1.81$ & 0.00 & 1.00 \\
\hline & Recessive model & & & Random & $81.4 \%$ & 0.00 & 0.80 & $0.53-1.23$ & 0.00 & 1.00 \\
\hline \multirow[t]{4}{*}{ Caucasian } & $\mathrm{BB} v s \mathrm{bb}$ & 978 & 1692 & Random & $78.3 \%$ & 0.00 & 0.78 & $0.41-1.47$ & 0.00 & 1.00 \\
\hline & $\mathrm{BB} v s \mathrm{Bb}$ & & & Fixed & $8.3 \%$ & 0.37 & 0.97 & $0.75-1.26$ & 0.00 & 1.00 \\
\hline & Dominant model & & & Random & $59.8 \%$ & 0.02 & 1.14 & $0.75-1.73$ & 0.00 & 1.00 \\
\hline & Recessive model & & & Random & $81.5 \%$ & 0.00 & 0.77 & $0.48-1.26$ & 0.00 & 1.00 \\
\hline \multirow[t]{4}{*}{ Asians } & $\mathrm{BB} v s \mathrm{bb}$ & 490 & 485 & Random & $88.8 \%$ & 0.00 & 0.92 & $0.14-6.14$ & 0.00 & 1.00 \\
\hline & $\mathrm{BB} v s \mathrm{Bb}$ & & & Random & $77.5 \%$ & 0.01 & 0.92 & $0.25-3.38$ & 0.00 & 1.00 \\
\hline & Dominant model & & & Random & $85.8 \%$ & 0.00 & 1.03 & $0.22-4.73$ & 0.00 & 1.00 \\
\hline & Recessive model & & & Random & $86.8 \%$ & 0.00 & 0.91 & $0.31-2.68$ & 0.00 & 1.00 \\
\hline Consistent with & $\mathrm{BB} v s \mathrm{bb}$ & 1040 & 1767 & Random & $82.3 \%$ & 0.00 & 0.78 & $0.41-1.50$ & 0.24 & 0.81 \\
\hline \multirow{3}{*}{ HWE } & $\mathrm{BB} v s \mathrm{Bb}$ & & & Fixed & $41.1 \%$ & 0.11 & 0.89 & $0.70-1.13$ & 0.24 & 0.81 \\
\hline & Dominant model & & & Random & $73.3 \%$ & 0.00 & 1.24 & $0.77-1.99$ & 0.24 & 0.81 \\
\hline & Recessive model & & & Random & $79.8 \%$ & 0.00 & 0.83 & $0.53-1.28$ & 0.24 & 0.81 \\
\hline
\end{tabular}

\section{Publication bias}

The publication bias of the meta-analysis for the association between the BsmI polymorphism and low BMD risk was detected using Begg's funnel plot; all graphical funnel 
plots of the included studies appeared to be symmetrical. The results of the Begg's funnel plot analyses are shown in Table 2 . The results revealed no publication bias (all $\mathrm{P}<0.05$ ).

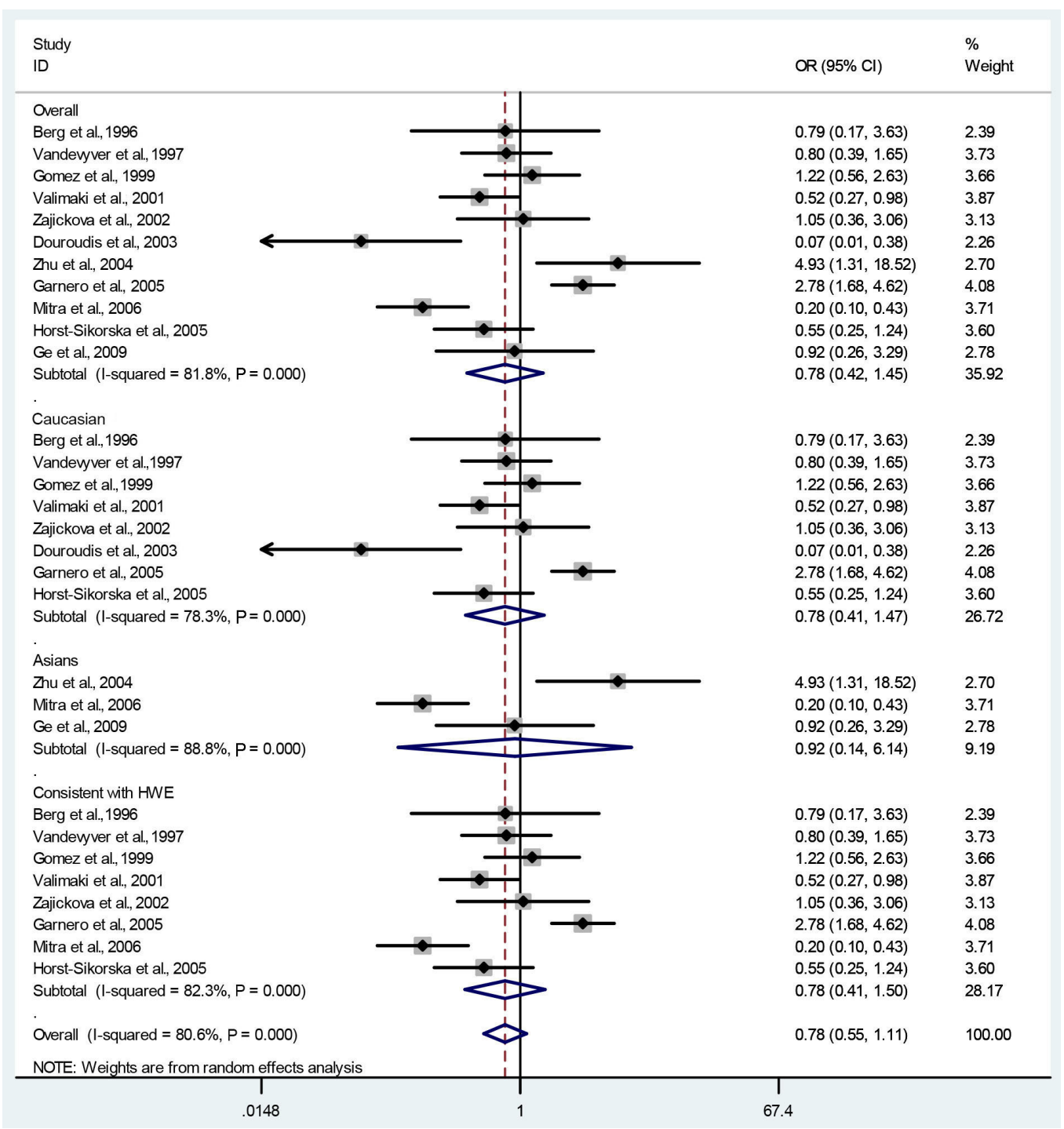

Figure 2. Association of vitamin D receptor Bsm I gene polymorphism with the risk for bone mineral density (BB vs bb).

\section{DISCUSSION}

There is growing evidence that genetic variation plays an important role in the determination of individual susceptibility to complex disease traits. Low BMD is a multifactorial 
disorder with a strong genetic component; the VDR gene has been suggested as a candidate gene for low BMD. The BsmI polymorphism is one of most important subtypes of VDR gene polymorphisms. In recent years, the association between the BsmI polymorphism and BMD has attracted increasing attention. Many studies have evaluated the association between the BsmI polymorphism of the VDR gene and low BMD risk in postmenopausal women, but the results are controversial, possibly because of variations in study design, small sample sizes, and heterogeneous populations. It is possible that the observed associations between the Bsm I polymorphism and low BMD risk reflect chance observations rather than true associations. To help clarify these inconsistent findings, we conducted this meta-analysis to obtain a competitive result via combining more eligible studies, enlarging the sample size, and conducting a subgroup analysis. A total of 28 case-control studies were included, representing 1468 low BMD cases and 2177 healthy controls. The results of our meta-analyses show that there is no evidence supporting an association between the BsmI polymorphism and the risk of low BMD. In the stratified analysis relating to ethnicity, our results indicated that the BsmI polymorphism was not associated with low BMD risk in Asians and in Caucasians. Deviations of allelic distributions from HWE may contribute to between-study heterogeneity; sensitivity analysis conducted by limiting this meta-analysis to studies that are consistent with HWE revealed that these results were realistic and reliable.

The function of the XRCC1 Arg280His polymorphism may be affected by gene-gene and gene-environment interactions. A previous study demonstrated that polymorphisms in both genes evaluated (BsmI polymorphism of the VDR gene and estrogen receptor genotypes) increased BMD risk, although the Bsm I polymorphism alone did not increase the risk of low BMD risk (Willing et al., 1998). In addition, obesity can mask the influence of the VDR genotypes on BMD, as demonstrated by Dawson-Hughes and Finneran, (1995). However, further studies of gene-gene and gene-environment interactions should be taken into consideration when assessing low BMD risk.

There were some limitations to our study. First, only published studies were included in this meta-analysis; thus, we cannot exclude the possibility of publication bias, although the results of statistical tests showed this to be unlikely. In addition, significant between-study heterogeneity was observed. Although we used the random effects model to pool ORs, this may have affected the precision of the results. Finally, although all cases and controls of each study were well defined with similar inclusion criteria, there may be potential factors that were not taken into account that influenced our results.

Despite these limitations, our meta-analysis has some advantages. This is the first meta-analysis of the relationship between the BsmI polymorphism of the VDR gene and low BMD risk in postmenopausal women. Notably, we established an effective search strategy based on computer-assisted and manual searching, and the quality of studies included in the current meta-analysis was satisfactory according to our selection criteria. In addition, explicit methods for study selection, data extraction, and data analysis were well designed before initiating the meta-analysis. Finally, there was no evidence of publication bias in this meta-analysis and the sensitivity analysis indicated that the results are statistically robust.

In conclusion, our study indicates that the VDR gene BsmI polymorphism is not associated with risk of low BMD in postmenopausal women. Gene-gene and gene-environment interactions should be investigated in further studies. 


\section{Conflicts of interest}

The authors declare no conflict of interest.

\section{ACKNOWLEDGMENTS}

This study was not funded by any Pharmaceutical Company.

\section{REFERENCES}

Berg JP, Falch JA and Haug E (1996). Fracture rate, pre- and postmenopausal bone mass and early and late postmenopausal bone loss are not associated with vitamin D receptor genotype in a high-endemic area of osteoporosis. Eur. J. Endocrinol. 135: 96-100.

Dawson-Hughes B and Finneran S (1995). Calcium absorption on high and low calcium intakes in relation to vitamin D receptor genotypes. J. Clin. Endocrinol. Metabol. 80: 3657-3661.

Douroudis K, Tarassi K, Ioannidis G, Giannakopoulos F, et al. (2003). Association of vitamin D receptor gene polymorphisms with bone mineral density in postmenopausal women of Hellenic origin. Maturitas 45: 191-197.

Gallagher JC, Fowler SE, Detter JR and Sherman SS (2001). Combination treatment with estrogen and calcitriol in the prevention of age-related bone loss. J. Clin. Endocrinol. Metab. 86: 3618-3628.

Garnero P, Munoz F, Borel O, Sornay-Rendu E, et al. (2005). Vitamin D receptor gene polymorphisms are associated with the risk of fractures in postmenopausal women, independently of bone mineral density. J. Clin. Endocrinol. Metab. 90: 4829-4835.

Ge JR, Xie LH, Chen K and Zeng XA (2009). Association of genetic polymorphisms in several vitamin D receptor gene sites with bone mineral density and biochemical markers of bone turnover in postmenopausal women. J. Clin. Rehab. Tissue Eng. Res. 13: 5593-5596.

Gomez C, Naves ML, Barrios Y, Diaz JB, et al. (1999). Vitamin D receptor gene polymorphisms, bone mass, bone loss and prevalence of vertebral fracture: differences in postmenopausal women and men. Osteoporos. Int. 10: 175-182.

Horst-Sikorska W, Wawrzyniak A, Celczyńska-Bajew L, Marcinkowska M, et al. (2005). Polymorphism of VDR gene-the most effective molecular marker of osteoporotic bone fractures risk within postmenopausal women from Wielkopolska region of Poland. Endokrynol. Pol. 56: 233-239.

Kanis JA, Melton LJ, III, Christiansen C, Johnston CC, et al. (1994). The diagnosis of osteoporosis. J. Bone Miner. Res. 9: $1137-1141$.

Mitra S, Desai M and Ikram KM (2006). Vitamin D receptor gene polymorphisms and bone mineral density in postmenopausal Indian women. Maturitas 55: 27-35.

Moore DD, Kato S, Xie W, Mangelsdorf DJ, et al. (2006). International Union of Pharmacology. LXII. The NR1H and NR1I receptors: constitutive androstane receptor, pregnene $\mathrm{X}$ receptor, farnesoid $\mathrm{X}$ receptor $\alpha$, farnesoid $\mathrm{X}$ receptor $\beta$, liver X receptor $\alpha$, liver X receptor $\beta$, and vitamin D receptor. Pharmacol. Rev. 58: 742-759.

Morrison NA, Qi JC, Tokita A, Kelly PJ, et al. (1994). Prediction of bone density from vitamin D receptor alleles. Nature 367: 284-287.

Pike JW, Yamamoto H and Shevde NK (2002). Vitamin D receptor-mediated gene regulation mechanisms and current concepts of vitamin D analog selectivity. Adv. Ren. Replace. Ther. 9: 168-174.

Qin G, Dong Z, Zeng P, Liu M, et al. (2013). Association of vitamin D receptor BsmI gene polymorphism with risk of osteoporosis: a meta-analysis of 41 studies. Mol. Biol. Rep. 40: 497-506.

Riggs BL (1997). Vitamin D-receptor genotypes and bone density. N. Engl. J. Med. 337: 125-126.

Seo HJ, Kim SG and Kim CS (2008). Risk factors for bone mineral density at the calcaneus in 40-59 year-old male workers: a cross-sectional study in Korea. BMC Public Health 8: 253.

Turner RT, Riggs BL and Spelsberg TC (1994). Skeletal effects of estrogen. Endocr. Rev. 15: 275-300.

Valimaki S, Tahtela R, Kainulainen K, Laitinen K, et al. (2001). Relation of collagen type I $\alpha 1$ (COLIA 1) and vitamin $\mathrm{D}$ receptor genotypes to bone mass, turnover, and fractures in early postmenopausal women and to hip fractures in elderly people. Eur. J. Intern. Med. 12: 48-56.

Vandevyver C, Wylin T, Cassiman JJ, Raus J, et al. (1997). Influence of the vitamin D receptor gene alleles on bone mineral density in postmenopausal and osteoporotic women. J. Bone Miner. Res. 12: 241-247.

Willing M, Sowers M, Aron D, Clark MK, et al. (1998). Bone mineral density and its change in white women: estrogen 
and vitamin D receptor genotypes and their interaction. J. Bone Miner. Res. 13: 695-705.

Zajickova K, Zofkova I, Bahbouh R and Krepelova A (2002). Vitamin D receptor gene polymorphisms, bone mineral density and bone turnover: FokI genotype is related to postmenopausal bone mass. Physiol. Res. 51: 501-509.

Zhu MJ, Yan XD, Wang F and Chen YH (2004). Relationship between vitamin D receptor genotypes and bone mineral density in Guangxi Zhuang and Han nationality postmenopausal women. Chin. J. Osteoporos. 10: 140-142. 